\title{
3 Research Square

\section{Long Non-Coding RNA FOXD1-AS1 Promotes the Progression and Glycolysis of Nasopharyngeal Carcinoma by Sustaining FOXD1 Expression}

\section{Zhanwang Wang}

Central South University Third Xiangya Hospital https://orcid.org/0000-0002-3655-0704

\section{Dong He}

Hunan Provincial People's Hospital

\section{Yuxing Zhu}

Central South University Third Xiangya Hospital

\section{Xueying Hu}

Central South University Third Xiangya Hospital

Yi Jin

Central South University Third Xiangya Hospital

\section{Lian Gong}

Central South University Third Xiangya Hospital

\section{Mengqing Xiao}

Central South University Third Xiangya Hospital

\section{Liang Xiang}

Central South University Third Xiangya Hospital

\section{Qinghai Zeng}

Central South University Third Xiangya Hospital

Jianye Liu

Central South University Third Xiangya Hospital

\section{Xingyu Chen}

Central South University Third Xiangya Hospital

\section{Yeyu Zhang}

Central South University Third Xiangya Hospital

Ke Cao ( $D$ csucaoke@163.com )

Central South University Third Xiangya Hospital

\section{Research}

Keywords: FOXD1-AS1, progression, glycolysis, nasopharyngeal carcinoma, FOXD1 
Posted Date: August 11th, 2020

DOI: https://doi.org/10.21203/rs.3.rs-52587/v1

License: (c) (i) This work is licensed under a Creative Commons Attribution 4.0 International License. Read Full License 


\section{Abstract}

Background: Increasing evidence have emphasized the importance of long non-coding RNAs (IncRNAs) in various human cancers progression. Forkhead box D1 antisense RNA1 (FOXD1-AS1) is a novel IncRNA and plays vital regulatory role in diverse biological processes of cancers. However, the biological function, molecular mechanism and clinical significance of FOXD1-AS1 in nasopharyngeal carcinoma is still unknown.

Methods: Comprehensive bioinformatics analysis and qRT-PCR was conducted to detect the expression level of FOXD1-AS1. Loss-of-function and gain-of-function experiments were performed to verify the functions of FOXD1-AS1 in proliferation, migration, invasion, apoptosis and glycolysis of nasopharyngeal carcinoma in vitro and in vivo. Further bioinformatics analysis and experiments were carried out to explore the underlying molecular mechanisms of FOXD1-AS1.

Results: FOXD1-AS1 was significantly overexpressed in nasopharyngeal carcinoma and associated with poor survival in patients. Knockdown of FOXD1-AS1 significantly inhibited cell proliferation, migration, invasion and glycolysis, and promotes apoptosis in nasopharyngeal carcinoma, whereas over-expression of FOXD1-AS1 has the opposite effect. Mechanistically, we found that FOXD1-AS1 could upregulate the expression of FOXD1 through stabilizing the FOXD1 expression at mRNA and protein levels, and FOXD1 increased the glycolysis level by transcriptionally upregulating the expression of LDHA, PKM and ENO1, thus playing an oncogenic role in nasopharyngeal carcinoma progression.

Conclusion: FOXD1-AS1 plays a critical regulatory role in nasopharyngeal carcinoma. The identified FOXD1-AS1/FOXD1 axis may serve as a potential prognostic biomarker and therapeutic target for patients with nasopharyngeal carcinoma.

\section{Background}

Nasopharyngeal carcinoma (NPC) is a highly invasive head and neck cancer that arises from the epithelial lining of the nasopharynx and is commonly found in Southeast Asia, especially China [1, 2]. Due to great improvements in magnetic resonance imaging, concurrent chemoradiotherapy, and intensitymodulated radiotherapy, the 5-year survival rate of NPC patients has substantially improved [3]. The majority of NPC patients, however, are diagnosed at stage III or IV, and $30 \%$ of patients with NPC eventually develop distant metastases and/or recurrence, thereby having far from satisfactory treatment results $[4,5]$. Due to these outcomes, it is critical to identify novel predictive biomarkers and therapeutic targets for individualized treatment to improve the clinical outcomes of patients.

Long noncoding RNAs (IncRNAs) are a novel class of RNAs that are characterized as being more than 200 bases in length without having protein-coding potential or encoding only a small peptide [6, 7]. Increasing evidence has revealed that IncRNAs are involved in various pathophysiological and physiological processes, such as cell proliferation, apoptosis, invasion, metastasis, and therapeutic resistance [8-11]. Although some studies have validated that IncRNAs play a vital role in the progression, 
metastasis, and occurrence of multiple human cancers, including NPC [12-16], how IncRNAs function in patients with NPC remains unclear. Thus, further investigation into IncRNA regulatory mechanisms to provide prognostic biomarkers and therapeutic targets for NPC patients would be beneficial. FOXD1 antisense RNA1 (FOXD1-AS1) is a novel IncRNA that has been reported to function as a potential oncogenic biomarker in glioma [17]. Nevertheless, the pivotal role of FOXD1-AS1 in NPC remains to be elucidated.

In this study, we found that FOXD1-AS1 was highly overexpressed in NPC compared to non-NPC (normal) tissues, and overexpression of FOXD1-AS1 was a predictor for poor overall survival (OS) and disease-free survival (DFS). In vitro and in vivo functional experiments verified that FOXD1-AS1 promotes NPC cell proliferation, migration, metastasis, and glycolysis levels, and inhibits apoptosis of NPC cells by upregulating FOXD1 expression. Therefore, our study elucidates the regulatory mechanism and clinical significance of FOXD1-AS1 in NPC and provides a potential prognostic biomarker and therapeutic target for NPC.

\section{Methods And Materials}

\section{Patients and specimens}

Nasopharyngeal carcinoma tissues and normal nasopharyngeal tissues were obtained from 10 NPC patients who received treatment at the Third Xiangya Hospital (Changsha, Hunan, China) from May 2017 to May 2019. The patients were diagnosed by pathological analysis and were not subjected to radiation therapy or chemotherapy. The Institutional Review Board of the Ethics Committee of Third Xiangya Hospital approved the consent procedure, and written informed consent was provided by all patients in the study.

\section{Cell culture and transfection}

Human NPC cells (CNE-1, CNE-2, HNE1, HNE2, 5-8F, and 6-10B) and nasopharyngeal epithelial cells (NP69) were obtained from American Type Culture Collection (ATCC). The cells were maintained in Dulbecco's Modified Eagle Medium (DMEM; Gibco, Carlsbad, CA, USA) supplemented with 10\% fetal bovine serum (FBS; Gibco, Carlsbad, CA, USA) and 1\% penicillin-streptomycin liquid (Life Technologies) in a humidified atmosphere at $37^{\circ} \mathrm{C}$ with $5 \% \mathrm{CO}_{2}$. ShRNAs and overexpression plasmids of FOXD1-AS1, FOXD1, and the negative control were designed by GeneChem Biotechnology Company (Shanghai, China). Lipofectamine 3000 (Invitrogen, Carlsbad, CA, USA) was used to transfect the recombinant plasmid according to the manufacturer's protocol as previously described [18]. Transfection efficiency was confirmed by quantitative reverse transcription polymerase chain reaction (qRT-PCR).

\section{Quantitative real-time PCR (qRT-PCR)}

A TRIzol reagent (Invitrogen) was used to extract total RNA from cells or tissues in accordance with the manufacturer's instructions. Approximately $500 \mathrm{ng}$ of total RNA was subjected to complementary DNA 
(cDNA) synthesis using a reverse transcription kit (Takara, Dalian, China). qRT-PCR was performed using an SYBR Green Premix Ex Taq kit (Takara, Dalian, China) on the ABI 7900HT qRT-PCR system (Life Technologies). $\beta$-actin was used as an internal control, and all experiments were performed at least three times. The $2^{-\triangle \Delta C t}$ method was used to calculate the relative expression levels of genes. The following primer sequences were used: FOXD1-AS1 -F: 5-CATAAATAGAGGG ACCCGCA-3; R: 5GGAAGAAGGTAGGAATCCCG-3'; FOXD1 -F: 5-TGAGCA CTGAGATGTCCGATG-3; R: 5CACCACGTCGATGTCT GTTTC-3'; $\beta$-actin -F: 5-CATGTACGTTGCTATCCAGGC-3; R: 5-CTCCTTAATGT CACGCACGAT-3'.

\section{MTT assays}

Approximately $1 \times 10^{4}$ NPC cells were seeded in a 96-well plate and incubated for $0,12,24,48$, and $72 \mathrm{~h}$. After the incubation period, $50 \mu \mathrm{L}$ MTT (Beyotime, Shanghai, China) was added into each well and reincubated in a $5 \% \mathrm{CO}_{2}$ atmosphere at $37^{\circ} \mathrm{C}$ for $4 \mathrm{~h}$. Subsequently, the MTT was discarded and $150 \mu \mathrm{L}$ of dimethyl sulfoxide (DMSO) was added into each well to dissolve the MTT crystals. Finally, the optical density at $570 \mathrm{~nm}$ was measured.

\section{Wound healing assays}

Wound healing assays were performed to assess the migration capacity of NPC cells as previously described. NPC cells $\left(1 \times 10^{5} / \mathrm{mL}\right)$ were cultivated in 6 -well plates. Once the cells were grown to $90 \%$ confluence, a wound was scratched using a $200-\mu l$ pipette tip, after which the cells were washed twice with phosphate-buffered saline (PBS) to dislodge any debris. DMEM without FBS was added to the 6-well plates. The photographs of wound healing were taken via a microscope at $0 \mathrm{~h}$ and $48 \mathrm{~h}$.

\section{Transwell assays}

Transwell assays were performed to assess the invasion of NPC cells. After transfection for $24 \mathrm{~h}$, NPC cells were collected and washed twice with serum-free DMEM. A $200 \mu \mathrm{l}\left(5 \times 10^{4}\right.$ cells $\left./ \mathrm{mL}\right)$ cell suspension was plated into the upper chamber, which was pre-coated with Matrigel Matrix (Corning, USA), while 600 $\mu \mathrm{l}$ DMEM containing $20 \%$ FBS was added to the lower transwell chamber. After incubation at $37^{\circ} \mathrm{C}$ for 24 h, cells that had invaded across the membrane were fixed with $95 \%$ ethanol for 20 min and then stained with hematoxylin for $10 \mathrm{~min}$. Images of stained cells were randomly photographed and counted under an inverted microscope.

\section{Apoptosis via flow cytometry}

The apoptosis rate of NPC cells was detected using an Annexin V-PE/7-AAD apoptosis detection kit (KA3809, Abnova) following the manufacturer's instructions. Briefly, approximately $3 \times 10^{5}$ cells were seeded into 6-well plates. After transfection with different shRNAs or plasmids for $48 \mathrm{~h}$, the cells were fully digested and collected. Cells were suspended in a cold binding buffer to a concentration of $1 \times 10^{6}$ 
cells $/ \mathrm{mL}$, and then stained with $5 \mu$ l of Annexin V-PE and $5 \mu \mathrm{L}$ of 7-AAD solution in the dark at room temperature. The results were observed via flow cytometry (Beckman Coulter, Inc.).

\section{Measurement of lactate production and glucose consumption.}

Lactate production and glucose consumption were measured as previously described [19]. For this, $5 \times 10^{5}$ cells were cultured and incubated in 6-well plates for $10 \mathrm{~h}$, after which the medium was discarded and the cells were incubated with a fresh medium for $8 \mathrm{~h}$. The levels of lactate and glucose were detected using an automatic biochemical analyzer (HITACHI, Tokyo, Japan). Consistent amounts of protein were used to normalize the relative levels of lactate production and glucose consumption.

\section{Western blotting}

Cells and/or tissues were washed twice with PBS, and RIPA lysis buffer containing 10\% protease inhibitor mixture (Roche, USA) was used to lyse cells and tissues. A bicinchoninic acid assay kit (Beyotime, Shanghai, China) was used to detect the protein concentration. For this, $30 \mu \mathrm{g}$ of protein was separated on an SDS-PAGE gel, and then transferred onto a polyvinylidene fluoride (PVDF) membrane. The PVDF membranes were blocked in $5 \%$ milk powder and then incubated overnight at $4^{\circ} \mathrm{C}$ with anti-FOXD1 (Omnimabs, OM203273), anti-LDHA (Ptgcn, 19987-1-AP), anti-PKM (Omnimabs, OM268791), anti-ENO1 (Ptgcn, 11204-1-AP), and anti- $\beta$-actin (Ptgcn, 66009-1-Ig). Membranes were incubated with secondary antibodies for $1 \mathrm{~h}$. The signals of protein bands were visualized by enhanced chemiluminescence and normalized using $\beta$-actin as an internal reference.

\section{Fluorescence in situ hybridization}

Fluorescence in situ hybridization (FISH) assays were performed to verify the location and expression of FOXD1-AS1 in NPC cells. Blue DAPI stain and red FISH Probe were purchased from RiboBio company (Guangzhou, China). A FISH kit (RiboBio, Guangzhou, China) was used to perform these assays in accordance with the manufacturer's protocols.

\section{Immunohistochemistry (IHC)}

Paraffin-embedded NPC tissues were sliced, dewaxed, hydrated, and antigen-repaired. Endogenous peroxidase was blocked; anti-FOXD1 (Omnimabs, OM203273), anti-Ki67 (Genetex, GTX16667), and antiCaspase 3 were added to these sections and incubated together at $4{ }^{\circ} \mathrm{C}$ overnight. Polymer enhancers were incubated for $20 \mathrm{~min}$ at room temperature, then biotin-labeled secondary antibodies were added and incubated for $30 \mathrm{~min}$ at room temperature. Next, a diaminobenzidine staining solution was used to stain the sections, followed by counterstaining with hematoxylin, and then the sections were mounted in glycerol-vinyl-alcohol (GVA Mount, Zymed). Two independent pathologists, who were blinded to the data and histopathological characteristics of the patients, evaluated the $\mathrm{IHC}$ scores according to the scoring standards as previously described.

RNA immunoprecipitation (RIP) 
The EZ-Magna RIP Kit (Millipore, USA) was used to perform the RIP assay. In brief, CNE-1 and 5-8F cells were collected and incubated with RIP lysis buffer. Subsequently, anti-FOXD1, anti-SNRNP70 antibody (positive control), and normal rabbit IgG (negative control) were added to the cell lysates. The acid phenol/chloroform method was used to isolate and purify RNA, followed by qRT-PCR for the detection of FOXD1-AS1 and FOXD1. The primers used were as follows: U1 -F: 5'-GGGAGATACCATGATCACGAAGGT-3'; R: 5'-CCACAA ATTA TGCAGTCGAGTTTCCC-3'; FOXD1-AS1 -F: 5'-CATAAATAGAGGGACCCGCA- 3'; R: 5'GGAAGAAGGTAGGAATCCCG-3'; FOXD1 -F: 5'-TGAGCACTGAGATG TCCGATG-3'; R: 5'CACCACGTCGATGTCTGTTTC-3'.

\section{Stability and a-amanitin treatment}

CNE-1 and 5-8F cells stably expressing shRNA against FOXD1-AS1 or NC were seeded into 6-well culture plates. Cells were then treated with $5 \mu \mathrm{g} / \mathrm{ml}$ actinomycin $\mathrm{D}$ and were harvested at the indicated time points. Total RNA was extracted for cDNA synthesis and subjected to qRT-PCR after $0,2,4,6,8$, and $12 \mathrm{~h}$ of treatment.

\section{RNA pull-down assay}

Pierce $^{\mathrm{TM} M}$ Magnetic RNA-Protein Pull-Down Kit (Thermo Fisher, Rockford, IL, USA) was used to perform RNA pull-down assays. Biotinylated bio-NC and bio-FOXD1-AS1 were transfected into CNE-1 and 5-8F cells. After being transfected for $48 \mathrm{~h}$, the cell lysates were mixed with magnetic beads to form proteinbio/RNA-magnetic bead complexes, and high-salt elution was used to obtain protein-bio/RNA complexes. Subsequently, western blotting was performed to measure the relative expression of FOXD1 in the protein-bio/RNA mixture.

\section{Chromatin immunoprecipitation (ChIP)}

ChIP assays were performed as previously described [11]. For this, protein-DNA complexes were obtained with the anti-FOXD1 antibody (1:50; Omnimabs, OM203273), followed by analysis of the resulting retrieved RNA subjected to qRT-PCR. The primers for qRT-PCR quantification were designed according to the promoter regions of FOXD1-AS1, lactate dehydrogenase A (LDHA), pyruvate kinase M2 (PKM) and enolase 1 (ENO1), which contain FOXD1 binding sites. The primer sequences used were as follows: FOXD1-AS1 -F: 5-ACAGATGGCGGAAAACAAA C-3; R: 5-GGATAAC CACCCCATCCTCT-3; LDHA -F: 5TTCACTGTGAGTGGG AGCTG-3; R: 5-CTCAGGAAGGCTT GGATCTG-3; PKM -F: 5-CCCAGCTCTGC GCTAATATC-3; R: 5-GGCCGTTTTCCTCTTAGGAC-3; ENO1 -F: 5-ACGGAATA TGACCCGTCTTG-3; R: 5CGTGCTTCCCCAGTGTTAAT-3; GAPDH -F: 5-TACT AGCGGTTTTACGGGCG-3; R: 5TCGAACAGGAGGAGCAGAGAGCGA-3'.

\section{Xenograft mouse model}

Female BALB/c nude mice, aged 6 weeks $(20 \pm 2 \mathrm{~g})$, were purchased from the Laboratory Animal Center of Central South University (Changsha, China) and subjected to tumor implantation. CNE-1 and 5-8F cells 
$\left(5 \times 10^{6} \mathrm{cells} / \mathrm{ml}, 0.2 \mathrm{ml}\right)$ were transfected with either shRNA targeting FOXD1-AS1, shRNA targeting FOXD1-AS1+FOXD1 overexpression plasmids, or vector plasmids, and were then subcutaneously injected into the mice. On day 25 , the mice were euthanized to harvest tumors, and the volume of the tumors was calculated using the formula $\mathrm{V}=\left(\mathrm{L} \times \mathrm{W}^{2}\right) / 2$. Tumors obtained from the mice were examined using routine hematoxylin and eosin (H\&E) staining and photographed under a light microscope. All experimental procedures were approved by the Ethics Committee of the Third Xiangya Hospital of Central South University (Changsha, China).

\section{Statistical analyses}

Data are presented as mean $\pm S D$, and all experiments were repeated independently three times. GraphPad Prism 8.0 Software (San Diego, CA, USA) was used to analyze the data. Student's t-tests or one-way ANOVA were used to analyze differences between groups. $\mathrm{P}<0.05$ (two-sided) was considered statistically significant.

\section{Results}

\section{Upregulated FOXD1-AS1 expression is negatively correlated with the prognosis of nasopharyngeal carcinoma}

Analysis of both GEPIA and GEO (GSE95166) databases revealed that FOXD1-AS1 was upregulated in multiple cancers (Figure S1A), including NPC (Fig. 1A, B). Using qRT-PCR, we further verified that the expression level of FOXD1-AS1 was higher in NPC tissues than in adjacent non-cancerous nasopharyngeal tissues (Fig. 1C). Patients with higher expression levels of FOXD1-AS1 had a shorter overall survival (OS; $P=0.017$, Fig. 1D) and a shorter disease-free survival (DFS; $P=0.03$, Fig. 1E) time. Taken together, these results suggest that upregulated FOXD1-AS1 expression may promote tumor progression and serve as a prognostic biomarker in patients with NPC.

\section{FOXD1-AS1 promotes proliferation, migration, and invasion, and inhibits apoptosis in NPC}

To investigate the functions of FOXD1-AS1 in NPC, we measured the expression levels of FOXD1-AS1 in several NPC cells (6-10B, HNE1, HNE2, CNE-1, CNE-2, and 5-8F), as well as in normal nasopharyngeal epithelial cells (NP69). We found that FOXD1-AS1 expression was higher in CNE-1 and 5-8F cells (Fig. 2A). Therefore, we chose these two cell lines to perform loss-of-function experiments by transfecting them with FOXD1-AS1-targeted shRNA1, shRNA2, shRNA3, and scramble control shRNA (NC). The FOXD1-AS1 expression level was detected by qRT-PCR, and the results indicated that FOXD1-AS1-targeted shRNA2 had the highest depletion efficiency (Fig. 2B). The FOXD1-AS1 over-expression plasmid was used to upregulate the expression level of FOXD1-AS1 in CNE-1 and 5-8F (Fig. 2C). The transfection efficiency of FOXD1-AS1 shRNA and over-expression plasmid are shown in Fig. 1F. Proliferation (Fig. 2D, E), migration (Fig. 2G, H), and invasion (Fig. 2I) were significantly inhibited when transfecting the FOXD1AS1-targeted shRNA2, whereas they were significantly increased when transfecting the FOXD1-AS1 overexpression plasmid. Furthermore, we observed that transfection with the FOXD1-AS1-targeted shRNA2 
could induce apoptosis, while transfection with the FOXD1-AS1 over-expression plasmid inhibited apoptosis of NPC cells (Fig. 2F). Together, these findings suggest that upregulation of FOXD1-AS1 significantly promotes the progression of NPC.

\section{FOXD1-AS1 upregulates FOXD1 expression}

To examine the detailed mechanism by which FOXD1-AS1 functions in patients with NPC, we conducted an RNA FISH assay, which indicated that FOXD1-AS1 is located in both the cytoplasm and nucleus of CNE-1 and 5-8F cells (Fig 3A). The UCSC Genome Browser reveals that FOXD1-AS1 is a divergent transcript of FOXD1; thus, we investigated their relationship in patients with NPC. We found that FOXD1 mRNA expression was positively correlated with FOXD1-AS1 mRNA upon analyzing GEPIA (Fig 3B) and ChIPBase (Fig S2A) databases. Furthermore, FOXD1 expression at the mRNA and protein levels decreased after FOXD1-AS1 depletion while increased under FOXD1-AS1 overexpression (Fig 3C, D). Therefore, we hypothesized that FOXD1-AS1 could upregulate the expression of FOXD1. First, CNE-1 and $5-8 \mathrm{~F}$ cells were transfected with NC or sh-FOXD1-AS1, followed by treatment with a-amanitin (an inhibitor of RNA polymerase II), which could stop new transcript synthesis. The results showed that FOXD1-AS1 depletion significantly shortened the half-life of FOXD1 mRNA (Fig 3E). Subsequently, FOXD1-AS1 was knocked down in CNE-1 and 5-8F cells following treatment with cycloheximide (CHX), a protein synthesis inhibitor, which could inhibit the synthesis of proteins. The results revealed that FOXD1-AS1 depletion strikingly reduced the stability of FOXD1 in CNE-1 and 5-8F cells (Fig 3F). Furthermore, it was verified that FOXD1-AS1 could directly bind to the FOXD1 protein by conducting an RNA pulldown assay (Fig 3G) and RIP assay (Fig 3H). Taken together, these results suggest that FOXD1-AS1 could upregulate the expression level of FOXD1.

\section{Elevated FOXD1 promotes proliferation, migration, and invasion, and suppresses apoptosis in NPC cells}

To study the function of FOXD1, stable FOXD1-depletion or FOXD1-overexpression cells were established, and the expression efficiency of FOXD1 was validated through qRT-PCR (Fig 4A, B). We then investigated the impact of FOXD1 on oncogenic potential, and the results revealed that FOXD1 significantly facilitated the proliferation (Fig 4C, D), migration (Fig 4F, G), and invasion (Fig 4H) of NPC cells. Furthermore, flow cytometry indicated that FOXD1-AS1 suppressed the apoptosis rate of CNE-1 and 5-8F cells (Fig 4E). Analysis of the GEPIA, Starbase, and GEO (GSE12452) databases revealed that FOXD1 expression was higher in NPC samples than in adjacent normal nasopharyngeal samples (Fig 5A-D), and higher levels of FOXD1 were correlated with shorter overall survival and disease-free survival times of patients with NPC (Fig 5E, F).

\section{FOXD1 promotes the glycolytic level of NPC cells}

To clarify the downstream pathway of FOXD1, KEGG analysis was performed using co-expression genes (top 200) of FOXD1 found in the GEPIA database, and the results showed that FOXD1 was associated with glycolysis (data not shown). Among multiple glycolysis-related genes found in the GEPIA and ChIPBase databases, we found that FOXD1 has a strong positive association with lactate dehydrogenase 
A (LDHA), pyruvate kinase M2 (PKM) and enolase 1 (ENO1) genes (Fig 5H and Fig S2B-D), which play vital role in glycolysis [20-22]. The protein levels of LDHA, PKM, and ENO1 significantly decreased after FOXD1 knockdown, whereas the levels increased under FOXD1 overexpression (Fig 5J). As a transcription factor, FOXD1 regulates the expression of specific genes. Analysis of UCSC and JASPAR databases revealed that FOXD1 could bind to the promoters of LDHA, PKM, ENO1, and FOXD1-AS1. We designed the primers according to the binding sites of these genes and conducted a CHIP assay, which verified that FOXD1 could in fact bind to the promoters of LDHA, PKM, and ENO1 (Fig 5I), and positively regulated their expression levels (Fig 5J), whereas FOXD1 could not bind to the promoter of FOXD1-AS1. To investigate the role of FOXD1 in anaerobic glycolysis, we transfected CNE-1 and 5-8F with FOXD1 silencing shRNA or FOXD1 overexpression plasmids, and biochemically detected ATP levels, glucose consumption, and lactic acid production. Knockdown of FOXD1 expression decreased ATP levels, glucose consumption, and lactic acid production in CNE-1 and 5-8F cells, and overexpression of FOXD1 increased the glycolytic metabolism of CNE-1 and 5-8F cells (Fig 5K). These results indicated that FOXD1 increases NPC cell glycolytic metabolism through transcriptionally regulated related glycolytic genes.

\section{FOXD1-AS1 promotes progression and glycolysis of NPC cells by sustaining FOXD1 expression}

To verify whether FOXD1-AS1 plays an oncogenic role in NPC by increasing FOXD1 expression, we transfected CNE-1 and 5-8F cells with FOXD1-AS1 silencing shRNA or FOXD1-AS1 silencing shRNA together with a FOXD1 overexpression plasmid. The depletion of FOXD1-AS1 decreased the protein levels of FOXD1, LDHA, PKM, and ENO1, as well as ATP levels, glucose consumption, and lactic acid production, whereas the overexpression of FOXD1 significantly reversed the effects of FOXD1-AS1 knockdown of proteins and glycolytic metabolism levels in CNE-1 and 5-8F cells (Fig 6A, B). MTT, flow cytometry, wound healing, and transwell assays all demonstrated that knockdown of FOXD1-AS1 expression in CNE-1 and $5-8 \mathrm{~F}$ cells inhibited proliferation, migration, and invasion, and induced apoptosis, whereas the overexpression of FOXD1 reversed this effect (Fig 6C-F).

\section{Effect of the FOXD1-AS1/FOXD1 axis on NPC cell progression in vivo}

Compared with normal tissues, FOXD1 was significantly increased in NPC tissues (Fig 7A), which was further verified by immunohistochemistry (Fig 7B). To confirm the effect of the FOXD1-AS1/FOXD1 axis in vivo, we employed a xenograft mouse model to validate it. The depletion of FOXD1-AS1 using the shFOXD1-AS1 plasmid reduced the tumorigenic ability of CNE-1 cells, whereas overexpression of FOXD1 reversed this effect (Fig 7C). Immunohistochemistry and western blot assays revealed that Ki67, caspase3, FOXD1, LDHA, PKM, and ENO1 protein levels were significantly decreased after knockdown of FOXD1-AS1 in CNE-1 cells, while these protein levels were restored by upregulating FOXD1 overexpression (Fig 7D, E).

\section{Discussion}

With great improvements technology, the therapeutic effect of radiotherapy on nasopharyngeal carcinoma becomes increasingly prominent, which significantly improves the prognosis and outcome of 
patients with NPC, $70-80 \%$ of whom have a 5-year overall survival rate [23]. However, some patients with NPC receiving radiotherapy develop radio-resistance, leading to local recurrence and distant metastasis. Simply increasing the radiation dose alone may cause serious post-treatment adverse reactions.

Therefore, further investigation of the critical molecules involved in the progression of NPC may provide a novel biomarker for improving the efficacy of radiotherapy in patients with NPC.

Accumulating evidence has shown that dysregulated IncRNAs play an important role in the progression of various human cancers, including NPC. For example, upregulation of FAM225A indicated poor survival of NPC and promoted tumorigenesis and metastasis by sponging miR-1275/miR-590-3p and upregulating ITGB3 expression [24]. AATBC facilitated migration, invasion, and metastasis of NPC cells in vitro and in vivo through the miR-1237-3p-PNN-ZEB1 axis [25]. In this study, we first reported that the IncRNA FOXD1-AS1 was obviously overexpressed in NPC tissues and cell lines and was closely correlated with poorer prognoses in patients with NPC. Therefore, it could be recognized as a prognostic biomarker for NPC. Functionally, the knockdown of FOXD1-AS1 inhibited cell proliferation, migration, and invasion, as well as promoted the apoptosis of NPC cells, revealing the oncogenic role of FOXD1-AS1. Similarly, the overexpression of FOXD1-AS1 contributed to cell growth, migration, and metastasis, and inhibited the apoptosis of NPC cells. Additionally, Gao et al. [17] reported that FOXD1-AS1 promotes cell proliferation and migration and decreases apoptosis by regulating elF5a protein levels in gliomas.

Besides FOXD1-AS1 upregulation, FOXD1 expression level is also significantly increased in NPC tissues and is associated with a worse OS and DFS in patients with NPC, implying that FOXD1 may be involved in NPC development and progression. As a transcription factor, the FOXD1 protein possesses a highly conserved DNA-binding domain composed of approximately 100 amino acids in a helix-turn-helix structure that could bind with specific DNA sequences on the promoter region of target genes, which promotes or inhibits the targeted gene expression [26-27]. Several studies have revealed that FOXD1 contributes to the progression of multiple cancers. For instance, increased FOXD1 expression facilitates cell proliferation and inhibits apoptosis in colorectal cancer by positively regulating Plk2, and a high expression of FOXD1 indicates a worse prognosis in these patients [28, 29]. Similarly, FOXD1 can function as a regulator of VEGF-A and promote angiogenesis in colorectal cancer [30]. Furthermore, FOXD1 promotes breast cancer cell growth by accelerating the transition from $\mathrm{G} 1$ phase to $S$ phase [31]. In our research, we demonstrated that ectopic expression of FOXD1 could promote cellular proliferation, migration, and invasion, and inhibit the apoptosis of NPC cells, suggesting that FOXD1 may serve as a potential therapeutic target for NPC.

To our knowledge, partial or natural antisense IncRNAs are generated from the antisense strand of a protein-coding gene and are involved in regulating the expression of this gene through various mechanisms [32-34]. The UCSC genome browser shows that FOXD1-AS1 is localized in the antisense strand of the FOXD1 gene, which prompted us to wonder whether FOXD1-AS1 might regulate FOXD1 expression to promote the progression and development of NPC. To verify this hypothesis, we examined the expression levels of FOXD1-AS1 and FOXD1, and we found that FOXD1-AS1 is concordantly overexpressed with FOXD1 in NPC cells and tissues. Furthermore, our results indicated that the silencing 
of FOXD1-AS1 contributed to the reduction of FOXD1 mRNA and protein levels, as well as to mRNA stability and protein degradation of FOXD1 in NPC cells. As such, FOXD1-AS1 and FOXD1 can form a socalled "RNA-RNA duplex" structure that protects FOXD1 mRNA from RNase degradation; moreover, we speculate that the mechanism via which FOXD1-AS1 stabilizes FOXD1 protein is that FOXD1-AS1 recruits deubiquitinating enzymes to avoid FOXD1 protein degradation by proteasomes, a hypothesis which needs to be further explored and validated in our future studies. Together, these results suggest that FOXD1-AS1 can stabilize FOXD1 mRNA and protein, thereby leading to the upregulation of FOXD1 expression at both RNA and protein levels. Similarly, several researchers also reported that antisense IncRNAs can regulate their related genes in cancer progression. For instance, EZR-AS1 promotes the mobility and invasiveness of esophageal squamous cell carcinoma via enhancing SMYD3-dependent H3K4 methylation which plays an important role in enhancing transcription of the EZR gene [35]. NAMPTAS promotes tumor progression and metastasis by serving as a sponge and recruiting transcription factors to epigenetically activate NAMPT in triple negative breast cancer [36]. The role of FOXD1-AS1 on the tumorigenic ability of NPC cells and the regulatory effect of FOXD1-AS1 on FOXD1 was also verified with in vivo experiments. Our findings suggest a potential molecular mechanism of FOXD1 upregulation in NPC.

Hypoxia is a fundamental characteristic of cancers and plays a vital role in the progression and development of human cancers [37]. Therefore, the glucose metabolism of tumor cells is different from that of normal cells. Tumor cells switch their metabolism mainly to glycolysis in an anaerobic environment, or even an environment with abundant oxygen (Warburg effect) [38]. The Warburg effect is also known as aerobic glycolysis, which explains the acceleration of tumor growth via increasing glucose uptake, ATP, and lactic acid production during the occurrence and progression of various human cancers [39]. Several studies have revealed that certain genes, including glucose transporter (GLUT), lactate dehydrogenase A (LDHA), hexokinase 2 (HK2), pyruvate kinase M2 (PKM), and enolase 1 (ENO1), are involved in glycolysis $[40,41]$. In this study, we found that FOXD1-AS1 could regulate the glycolysis of NPC cells by increasing the expression of LDHA, PKM, and ENO1 through the FOXD1-AS1-FOXD1 axis. These three glycolysis genes also indicated poor survival rates of NPC patients.

\section{Conclusions}

In summary, we demonstrated that FOXD1-AS1 is overexpressed in NPC cells and tissues, which is closely associated with the progression and poor prognosis in NPC patients. Furthermore, mechanistic research revealed that FOXD1-AS1 plays an oncogenic role in progression and promotes the glycolysis of NPC by sustaining the expression of FOXD1. The identified FOXD1-AS1 may serve as a potential prognostic biomarker and therapeutic target for patients with NPC.

\section{Abbreviations}

IncRNAs: Long non-coding RNAs; NPC: Nasopharyngeal carcinoma; FOXD1-AS1: forkhead box D1 antisense RNA1; OS: Overall survival; DFS: Disease-free survival; ATCC: American Type Culture Collection; 
DMEM: Dulbecco's Modified Eagle Media; qRT-PCR: Quantitative real-time PCR; cDNA: complementary DNA; PBS: Phosphate-buffered saline; PVDF: Polyvinylidene fluoride; TCGA: The Cancer Genome Atlas; GEPIA: Gene Expression Profiling Interactive Analysis; GEO: Gene Expression Omnibus; UCSC: University of California, Santa Cruz Genomics Institute; FISH: Fluorescence in situ hybridization; IHC:

Immunohistochemistry; RIP: RNA immunoprecipitation; ChIP: Chromatin immunoprecipitation; LDHA:

Lactate dehydrogenase A; PKM: Pyruvate kinase M2; ENO1: Enolase 1; HNSCC: Head and neck squamous cell carcinoma.

\section{Declarations}

\section{Acknowledgements}

We thank all the authors for their excellent work.

\section{Authors' contributions}

WZW, DH and KC designed the experiment, wrote the manuscript draft and analyzed the date. $X Y H, Y X Z$, YJ and LG contributed to data acquisition, interpretation and analysis. MQX, YH, YXC, YYZ, LPD, RRZ and JWX carried out the experiments. XYC performed all bioinformatics analysis. QHZ and JYL provide technical expertise and support. All authors have read and approved the final version of the manuscript.

\section{Funding}

This work was supported by the National Natural Science Foundation of China (81874137), the Outstanding Youth Foundation of Hunan Province (2018JJ1047), the Huxiang Young Talent Project (2016RS3022), the Hunan Province Science and technology talent promotion project (2019TJ-Q10), the Project of scientific research plan of health and Family Planning Commission of Hunan Province (c20180476), and the Fundamental Research Funds for the Central South University, Grant/Award Number: $2018 z z t s 938$.

\section{Availability of data and materials}

All data in our study are available upon request.

\section{Ethics approval and consent to participate}

This research was approved by the Ethics Committee of Third Xiangya Hospital of Central South University, and written informed consents were obtained before any operation to patients. The authors confirmed that we have obtained written consent from the patients to publish this manuscript.

\section{Consent for publication}

All authors have read the full article and agree to publish. 


\section{Competing interests}

The authors declare no conflicts of interest.

\section{References}

1. Lo KW, Chung GT, To KF. Deciphering the molecular genetic basis of NPC through molecular, cytogenetic, and epigenetic approaches. Semin Cancer Biol. 2012;22:79-86.

2. Wei KR, Zheng RS, Zhang SW, Liang ZH, Li ZM, Chen WQ. Nasopharyngeal carcinoma incidence and mortality in China, 2013. Chin J Cancer. 2017;36(1):90.

3. Blanchard P, Lee A, Marguet S, Leclercq J, Ng WT, Ma J, Chan AT, Huang PY, Benhamou E, Zhu G, et al. Chemotherapy and radiotherapy in nasopharyngeal carcinoma: an update of the MAC-NPC metaanalysis. Lancet Oncol. 2015;16: 645-55.

4. Lin JC, Jan JS, Hsu CY, Liang WM, Jiang RS, Wang WY. Phase III study of concurrent chemoradiotherapy versus radiotherapy alone for advanced nasopharyngeal carcinoma: positive effect on overall and progression-free survival. J Clin Oncol. 2003;21(4):631-37.

5. You R, Cao YS, Huang PY, Chen L, Yang Q, Liu YP, Zou X, Zhang YN, Jiang R, Zhang MX, et al. The Changing Therapeutic Role of Chemo-radiotherapy for Loco-regionally Advanced Nasopharyngeal Carcinoma from Two/ Three-Dimensional Radiotherapy to Intensity-Modulated Radiotherapy: A Network Meta-Analysis. Theranostics. 2017;7:4825-35.

6. Quinn JJ, Chang HY. Unique features of long non-coding RNA biogenesis and function. Nat Rev Genet. 2016;17:47-62.

7. Yao RW, Wang Y, Chen LL. Cellular functions of long noncoding RNAs. Nat Cell Biol. 2019;21:542-51.

8. Zhang E, Han L, Yin D, He XZ, Hong LZ, Si XX, Qiu MT, Xu TP, De W, Xu L, et al. H3K27 acetylation activated-long non-coding RNA CCAT1 affects cell proliferation and migration by regulating SPRY4 and HOXB13 expression in esophageal squamous cell carcinoma. Nucleic Acids Res. 2017;45:3086101.

9. Zhan Y, Zhang L, Yu S, Wen J, Liu Y, Zhang X. Long non-coding RNA CASC9 promotes tumor growth and metastasis via modulating FZD6/Wnt/ $\beta$-catenin signaling pathway in bladder cancer. $J$ Exp Clin Cancer Res. 2020;39:136.

10. He W, Zhong G, Jiang N, Wang B, Fan XX, Chen CH, Chen X, Huang J, Lin TX. Long noncoding RNA BLACAT2 promotes bladder cancer-associated lymphangiogenesis and lymphatic metastasis. J Clin Invest. 2018;128:861-75.

11. Zhu Y, He D, Bo H, Zexian Liu, Xiao MQ, Xiang L, Zhou JD, Liu Y, Liu XM, Gong L, et al. The MRVI1AS1/ATF3 signaling loop sensitizes nasopharyngeal cancer cells to paclitaxel by regulating the Hippo-TAZ pathway. Oncogene. 2019;38:6065-81.

12. Poliseno L, Salmena L, Zhang J, Carver B, Haveman WJ, Pandolfi PP. A coding-independent function of gene and pseudogene mRNAs regulates tumour biology. Nature. 2010;465:1033-38. 
13. Mercer TR, Dinger ME, Mattick JS. Long non-coding RNAs: insights into functions. Nat Rev Genet. 2009;10:155-9.

14. Huarte M. The emerging role of IncRNAs in cancer. Nat Med. 2015;21:1253-61.

15. Seiler J, Breinig M, Caudron-Herger M, Polycarpou-Schwarz M, Boutros M, Diederichs S. The IncRNA VELUCT strongly regulates viability of lung cancer cells despite its extremely low abundance. Nucleic Acids Res. 2017;45:5458-69.

16. Wen X, Tang X, Li Y, Xianyue Ren, Qingmei He, Xiaojing Yang, Jian Zhang, Yaqin Wang, Jun Ma, Na Liu. Microarray Expression Profiling of Long Non-Coding RNAs Involved in Nasopharyngeal Carcinoma Metastasis. Int J Mol Sci. 2016;17: 1956.

17. Gao YF, Liu JY, Mao XY, He ZW, Zhu T, Wang ZB, Li X, Yin JY, Zhang W, Zhou HH, et al. LncRNA FOXD1-AS1 acts as a potential oncogenic biomarker in glioma. CNS Neurosci Ther. 2020;26:66-75.

18. Zeng Q, Liu J, Cao P, Li JJ, Liu XM, Fan XJ, Liu L, Cheng Y, Xiong W, Li JG, et al. Inhibition of REDD1 Sensitizes Bladder Urothelial Carcinoma to Paclitaxel by Inhibiting Autophagy. Clin Cancer Res. 2018;24:445-59.

19. Li W, Peng C, Lee MH, Lim DY, Zhu F, Fu Y, Yang G, Sheng YQ, Xiao LB, Dong X, et al. TRAF4 is a critical molecule for Akt activation in lung cancer. Cancer Res. 2013;73:6938-50.

20. Wang C, Li Y, Yan S, Wang H, Shao XF, Xiao MM, Yang BC, Qin GX, Kong RR, Chen RB, et al. Interactome analysis reveals that IncRNA HULC promotes aerobic glycolysis through LDHA and PKM2. Nat Commun. 2020;11:3162.

21. Cai H, Li J, Zhang Y, Liao Y, Zhu Y, Wang C, Hou J. LDHA Promotes Oral Squamous Cell Carcinoma Progression Through Facilitating Glycolysis and Epithelial-Mesenchymal Transition. Front Oncol. 2019;9:1446.

22. Chen S, Zhang Y, Wang H, Zeng YY, Li Z, Li ML, Li FF, You J, Zhang ZM, Tzeng CM. WW domainbinding protein 2 acts as an oncogene by modulating the activity of the glycolytic enzyme ENO1 in glioma. Cell Death Dis. 2018;9:347.

23. Wei F, Wu Y, Tang L, Xiong F, Guo C, Li XY, Zhou M, Xiang B, Li XL, Li GY, et al. Trend analysis of cancer incidence and mortality in China. Sci China Life Sci. 2017;60:1271-75.

24. Zheng ZQ, Li ZX, Zhou GQ, Lin L, Zhang LL, Lv JW, Huang XD, Liu RQ, Chen FP, He XJ, et al. Long Noncoding RNA FAM225A Promotes Nasopharyngeal Carcinoma Tumorigenesis and Metastasis by Acting as ceRNA to Sponge miR-590-3p/miR-1275 and Upregulate ITGB3. Cancer Res. 2019;79:461226.

25. Tang T, Yang L, Cao Y, Wang MN, Zhang SS, Gong ZJ, Xiong F, He Y, Zhou YJ, Liao QJ, et al. LncRNA AATBC regulates Pinin to promote metastasis in nasopharyngeal carcinoma [published online ahead of print, 2020 May 4]. Mol Oncol. 2020.

26. Carlsson P, Mahlapuu M. Forkhead transcription factors: key players in development and metabolism. Dev Biol. 2002;250:1-23.

27. Li CH, Chang YC, Hsiao M, Liang SM. FOXD1 and Gal-3 Form a Positive Regulatory Loop to Regulate Lung Cancer Aggressiveness. Cancers (Basel). 2019;11:1897. 
28. Nakano I. Transcription factors as master regulator for cancer stemness: remove milk from fox?. Expert Rev Anticancer Ther. 2014;14:873-5.

29. Han T, Lin J, Wang Y, Fan QH, Sun HJ, Tao YM, Sun CX. Forkhead box D1 promotes proliferation and suppresses apoptosis via regulating polo-like kinase 2 in colorectal cancer. Biomed Pharmacother. 2018;103:1369-75.

30. Chen C, Xu ZQ, Zong YP, Ou BC, Shen XH, Feng H, Zheng MH, Zhao JK, Lu AG. CXCL5 induces tumor angiogenesis via enhancing the expression of FOXD1 mediated by the AKT/NF-KB pathway in colorectal cancer. Cell Death Dis. 2019;10:178.

31. Chang S, Sun L, Feng G. SP1-mediated long noncoding RNA POU3F3 accelerates the cervical cancer through miR-127-5p/FOXD1. Biomed Pharmacother. 2019;117:109133.

32. Engreitz JM, Haines JE, Perez EM, Munson G, Chen J, Kane M, McDonel PE, Guttman M, Lander ES. Local regulation of gene expression by IncRNA promoters, transcription and splicing. Nature. 2016;539:452-5.

33. Wery M, Gautier C, Descrimes M, Yoda M, Rendos HV, Migeot V, Gautheret D, Hermand D, Morillon A. Native elongating transcript sequencing reveals global anti-correlation between sense and antisense nascent transcription in fission yeast. RNA. 2018;24:196-208.

34. Su W, Xu M, Chen X, Chen N, Gong J, Nie L, Li L, Li XL, Zhang MN, Zhou Q. Long noncoding RNA ZEB1-AS1 epigenetically regulates the expressions of ZEB1 and downstream molecules in prostate cancer. Mol Cancer. 2017; 16:142.

35. Zhang XD, Huang GW, Xie YH, He JZ, Guo JC, Xu XE, Liao LD, Xie YM, Song YM, Li EM, et al. The interaction of IncRNA EZR-AS1 with SMYD3 maintains overexpression of EZR in ESCC cells. Nucleic Acids Res. 2018;46:1793-1809.

36. Zhang H, Zhang N, Liu Y, Su P, Liang YR, Li YM, Wang XL, Chen T, Song XJ, Sang YT, et al. Epigenetic Regulation of NAMPT by NAMPT-AS Drives Metastatic Progression in Triple-Negative Breast Cancer. Cancer Res. 2019;79:3347-59.

37. Bosco MC, D'Orazi G, Del Bufalo D. Targeting hypoxia in tumor: a new promising therapeutic strategy. J Exp Clin Cancer Res. 2020;39:43.

38. Hanahan D, Weinberg RA. Hallmarks of cancer: the next generation. Cell. 2011;144:646-74.

39. Wikoff WR, Grapov D, Fahrmann JF, DeFelice B, Rom WN, Pass HI, Kim K, Nguyen UT, Taylor SL, Gandara DR, et al. Metabolomic markers of altered nucleotide metabolism in early stage adenocarcinoma. Cancer Prev Res (Phila). 2015;8:410-8.

40. Li XB, Gu JD, Zhou QH. Review of aerobic glycolysis and its key enzymes- new targets for lung cancer therapy. Thorac Cancer. 2015;6:17-24.

41. Courtnay R, Ngo DC, Malik N, Ververis K, Tortorella SM, Karagiannis TC. Cancer metabolism and the Warburg effect: the role of HIF-1 and PI3K. Mol Biol Rep. 2015;42:841-51.

\section{Figures}


A

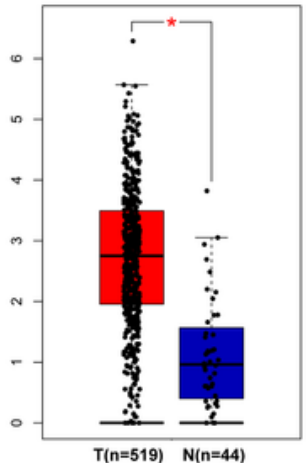

D

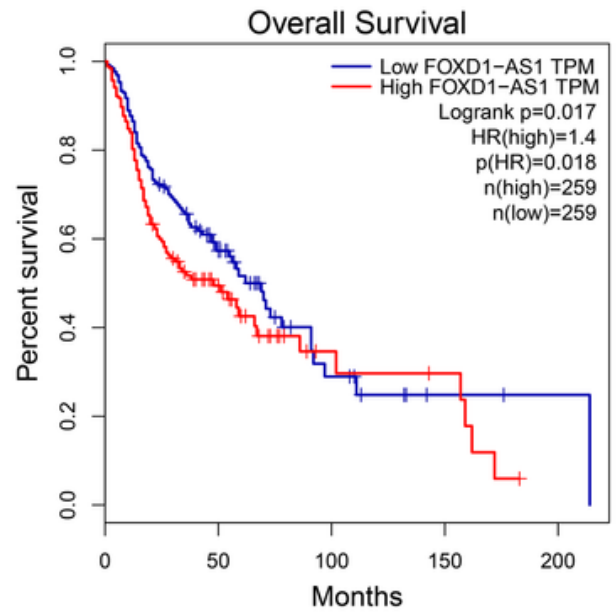

F

CNE-1

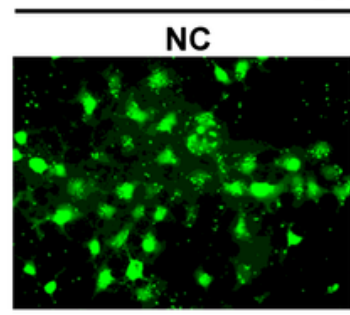

Ctr

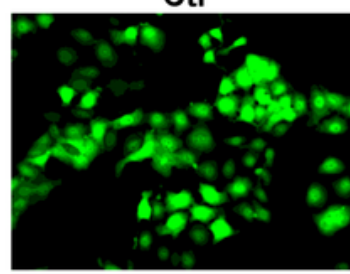

B

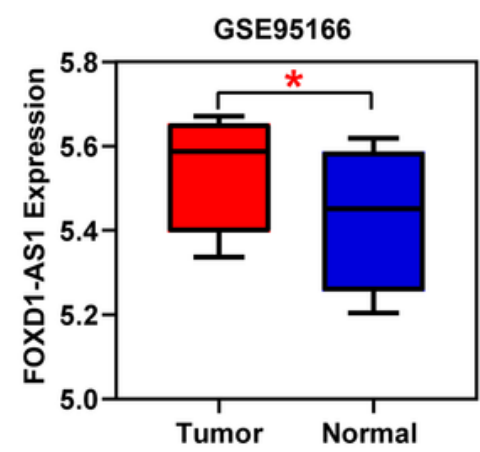

C

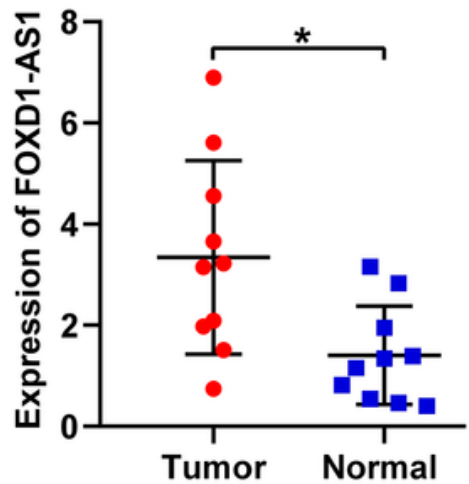

E

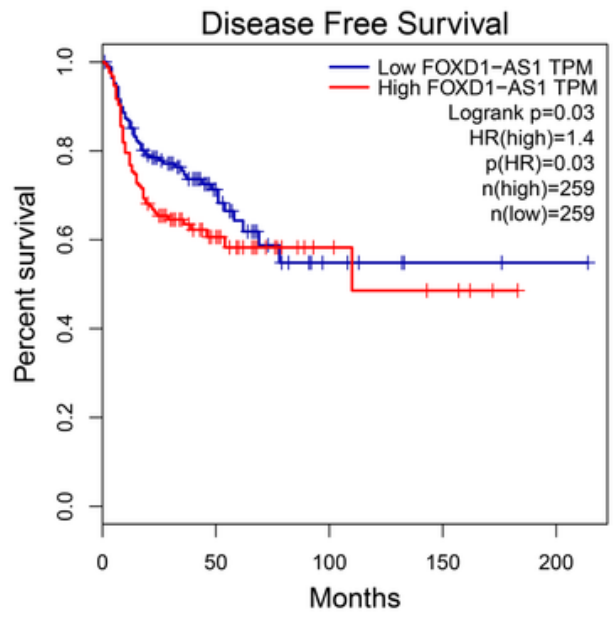

5-8F

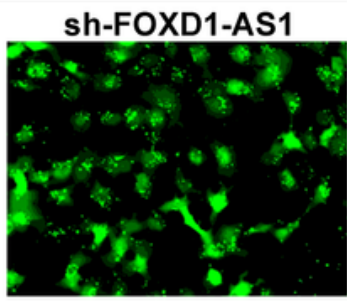

FOXD1-AS1

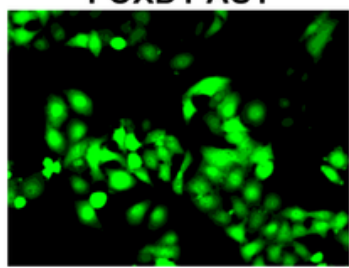

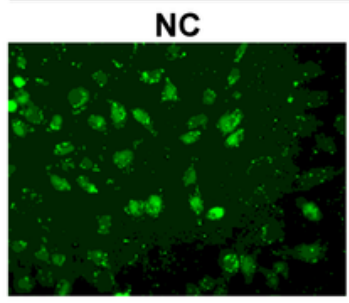

Ctr

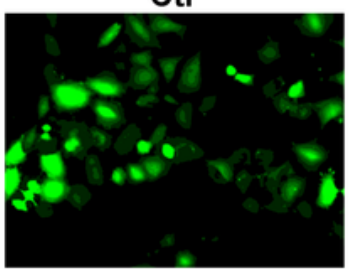

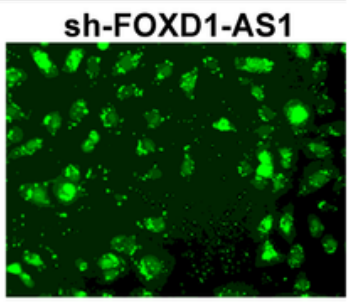

FOXD1-AS1

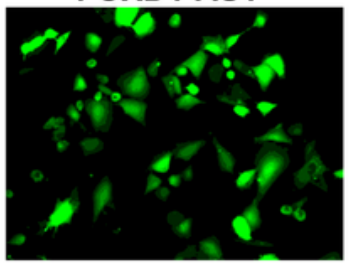

Figure 1

FOXD1-AS1 is overexpressed in NPC and is associated with poor prognosis in patients with NPC. (A) Review of the GEPIA database showed that FOXD1-AS1 is upregulated in multiple cancers. As seen in the GEPIA database (A) and the GSE95166 dataset (B), the expression level of FOXD1-AS1 is higher in tumor tissues than it is in normal tissues. (C) Further qRT-PCR testing confirms that FOXD1-AS1 was overexpressed in 10 pairs of NPC tissues and their adjacent normal tissues. Review of the GEPIA 
database showed that upregulation of FOXD1-AS1 is correlated with a shorter overall survival time (D) and disease-free survival time (E). (F) The transfection efficiency of FOXD1-AS1 shRNA and overexpression plasmid. Bars represent the standard deviation, $n s P>0.05,{ }^{\star} P<0.01, * \star P<0.001, * \star * P<$ 0.001.
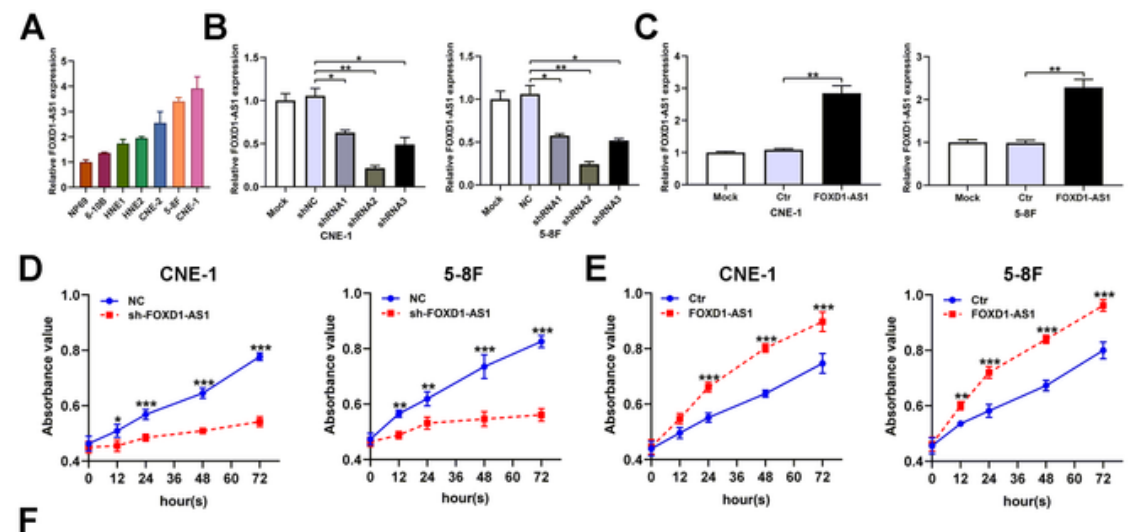

F

Apoptosis
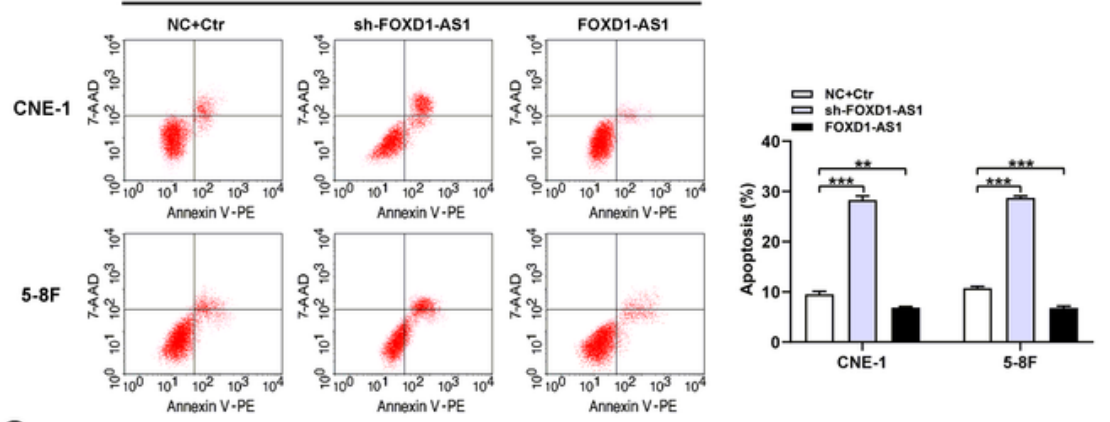

G

Migration
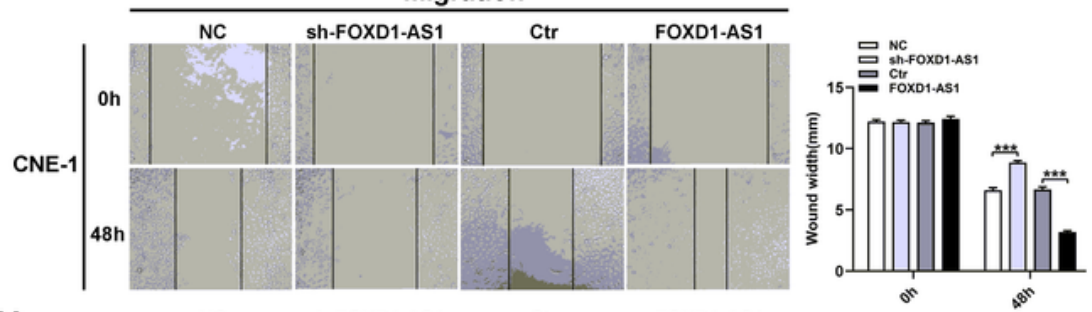

H
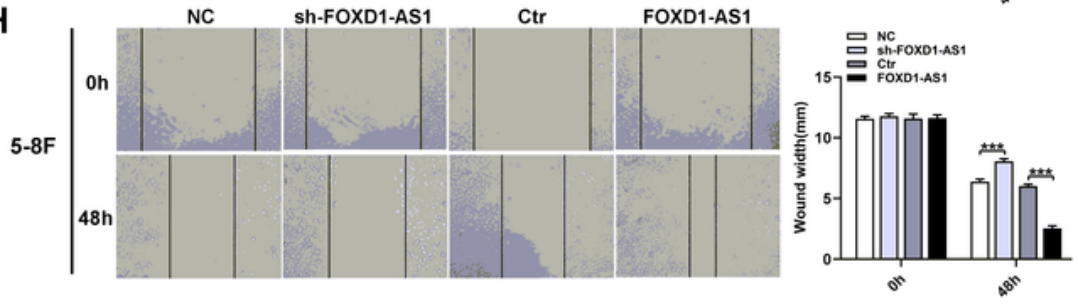

I

Invasion

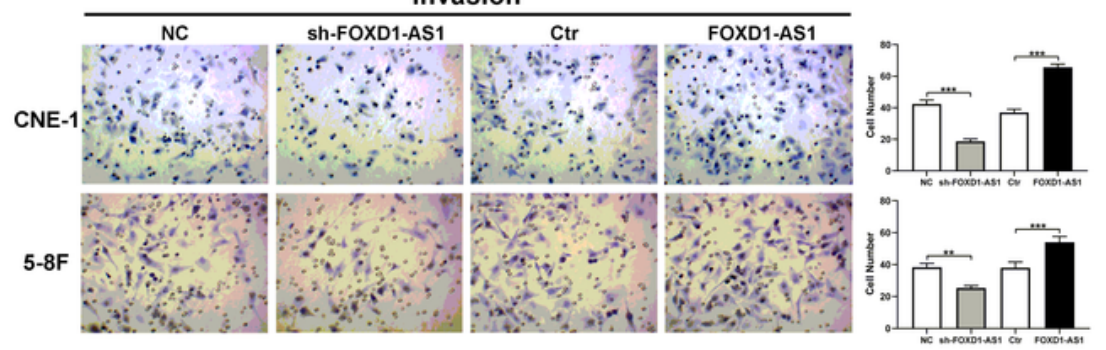

Figure 2 
FOXD1-AS1 promotes cell proliferation, migration, invasion, and inhibits apoptosis in NPC cells. (A) The expression levels of FOXD1-AS1 in various NPC cells (CNE-1, CNE-2, HNE-1, HNE-2, 5-8F, and 6-10B) and normal nasopharyngeal epithelial cells (NP69) were detected by qRT-PCR. (B) CNE-1 and 5-8F cells were transfected with FOXD1-AS1-target shRNA1, shRNA2, shRNA3, and scramble control shRNA (NC), respectively, and expression levels of FOXD1-AS1 were detected by qRT-PCR. (C) CNE-1 and 5-8F cells were transfected with FOXD1-AS1 overexpression plasmids or control (blank) plasmids for 48 hours, and qRT-PCR was used to analyze the efficiency. (D-E) MTT assay showing the effects of FOXD1-AS1 knockdown or overexpression on the cell proliferation of CNE-1 and 5-8F cells. (F) The effects of FOXD1AS1 knockdown and FOXD1-AS1 overexpression on the apoptosis of NPC cells were analyzed via flow cytometry assay. $(\mathrm{G}-\mathrm{H})$ Wound healing assay was conducted to assess the cell migration of $\mathrm{CNE}-1$ and 5-8F cells treated with knockdown or overexpression of FOXD1-AS1. (I) The invasion ability was significantly induced by FOXD1-AS1 knockdown, whereas it was obviously increased by FOXD1-AS1 overexpression. Bars represent standard deviation, ns $P>0.05,{ }^{*} P<0.01, * * P<0.001,{ }^{\star \star \star} P<0.001$. 
A

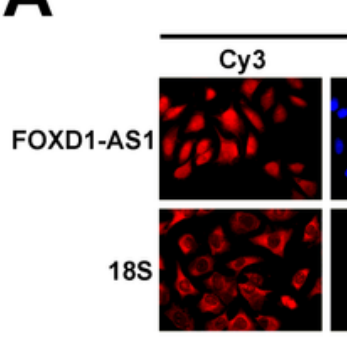

CNE-1
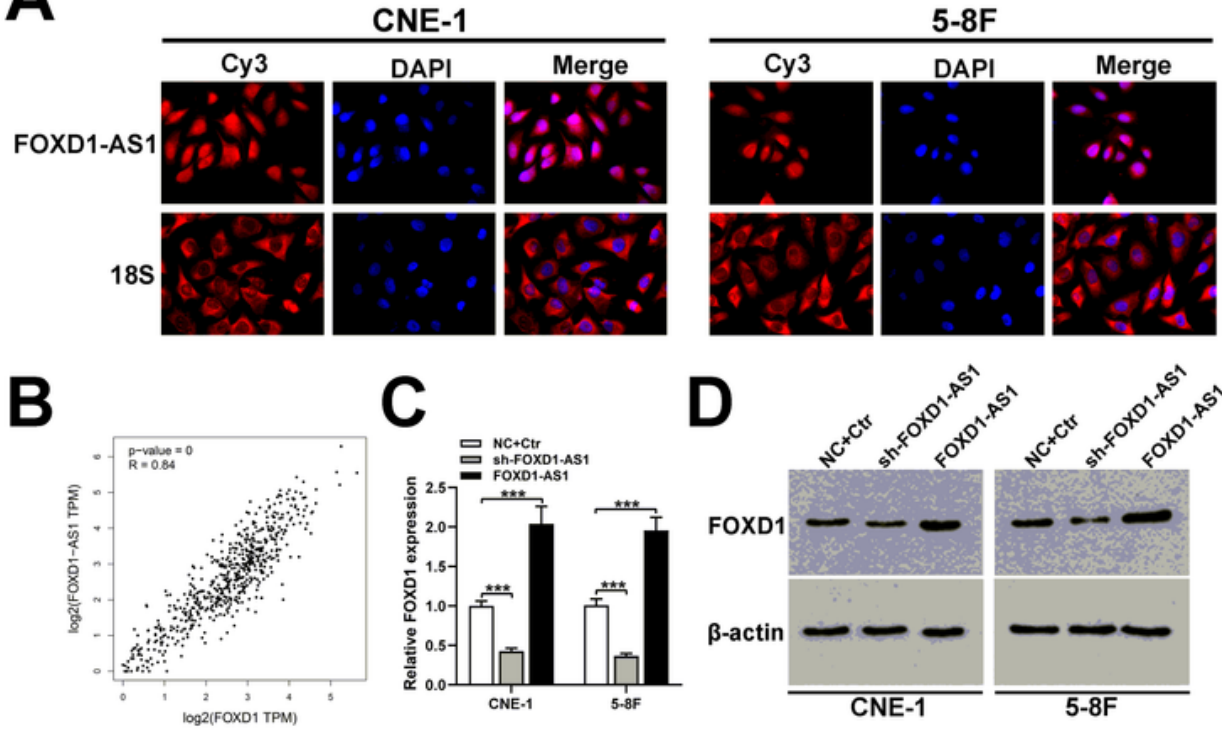

$\mathbf{E}$
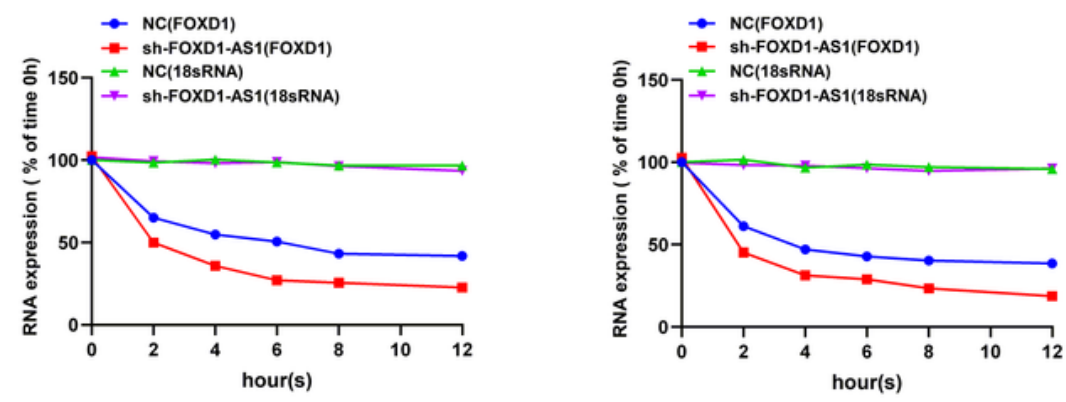

$\mathbf{F}$

CNE-1
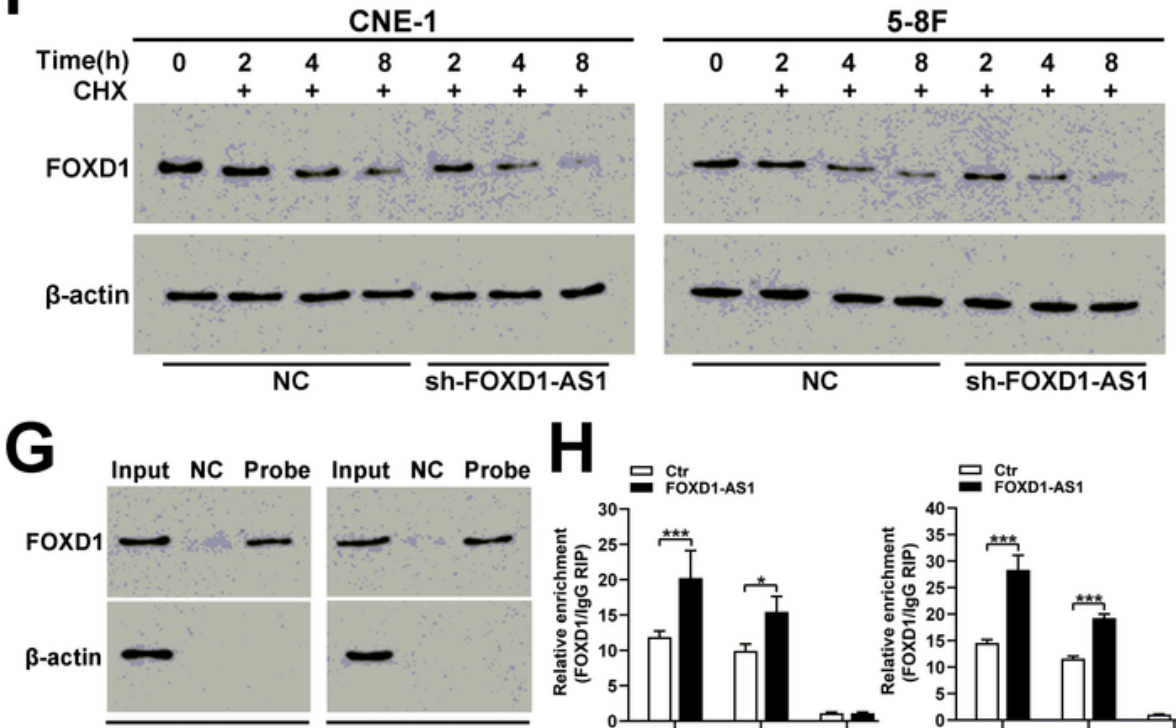

CNE-1
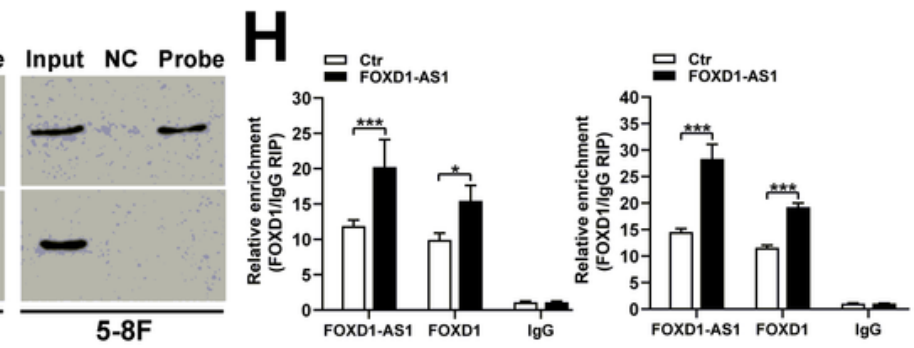

\section{Figure 3}

FOXD1-AS1 up-regulates FOXD1 expression at mRNA and protein levels. (A) FISH assay exhibiting that FOXD1-AS1 was simultaneously distributed in the cytoplasm and nucleus of CNE-1 and 5-8F cells. (B) FOXD1-AS1 is positively correlated with FOXD1 by analysis of GEPIA database. (C-D) qRT-PCR and western blot assay indicating that FOXD1-AS1 knockdown decreases the expression level of FOXD1, whereas FOXD1-AS1 overexpression increases the expression level of FOXD1. (E) RNA stability assay 
showing that FOXD1-AS1 depletion could significantly shorten the half-life of FOXD1 mRNA. (F) Protein stability assay revealing that FOXD1-AS1 knockdown promotes the degradation of FOXD1 protein. (G) RNA pull-down assay suggesting that FOXD1 binds with FOXD1-AS1. (H) RIP assay to verify that FOXD1AS1 binds to FOXD1. Bars represent standard deviation, ns $P>0.05$, ${ }^{*} P<0.01$, ${ }^{\star *} P<0.001$, $* \star * P<0.001$.

A
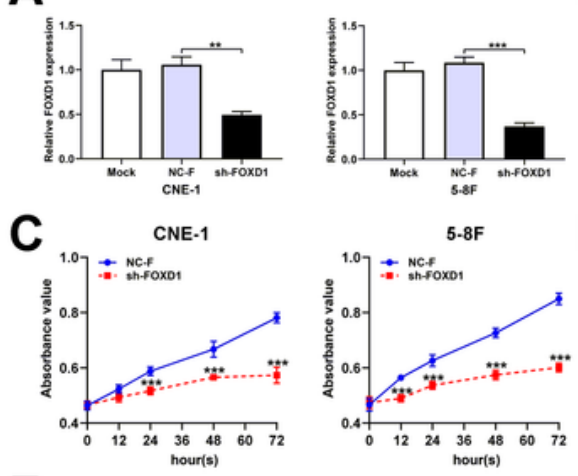

Apoptosis

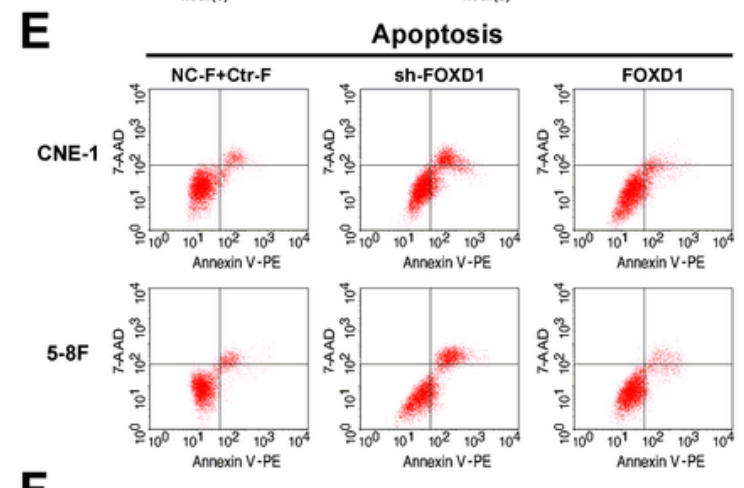

B
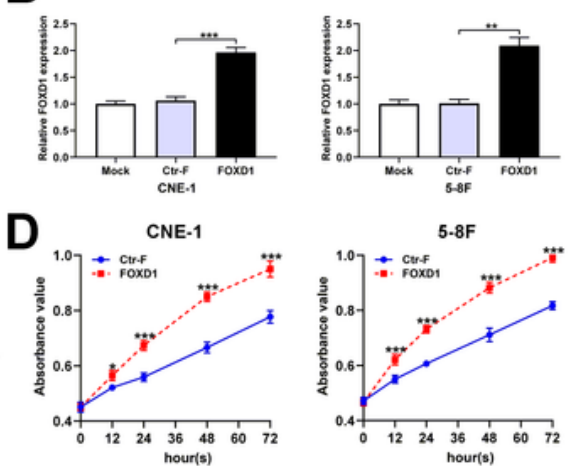

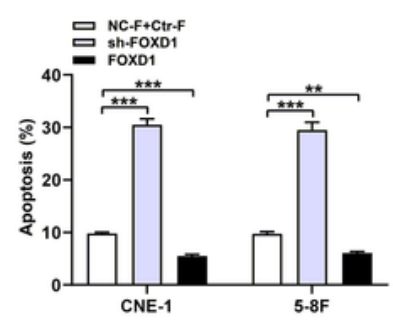

$\mathbf{F}$

Migration
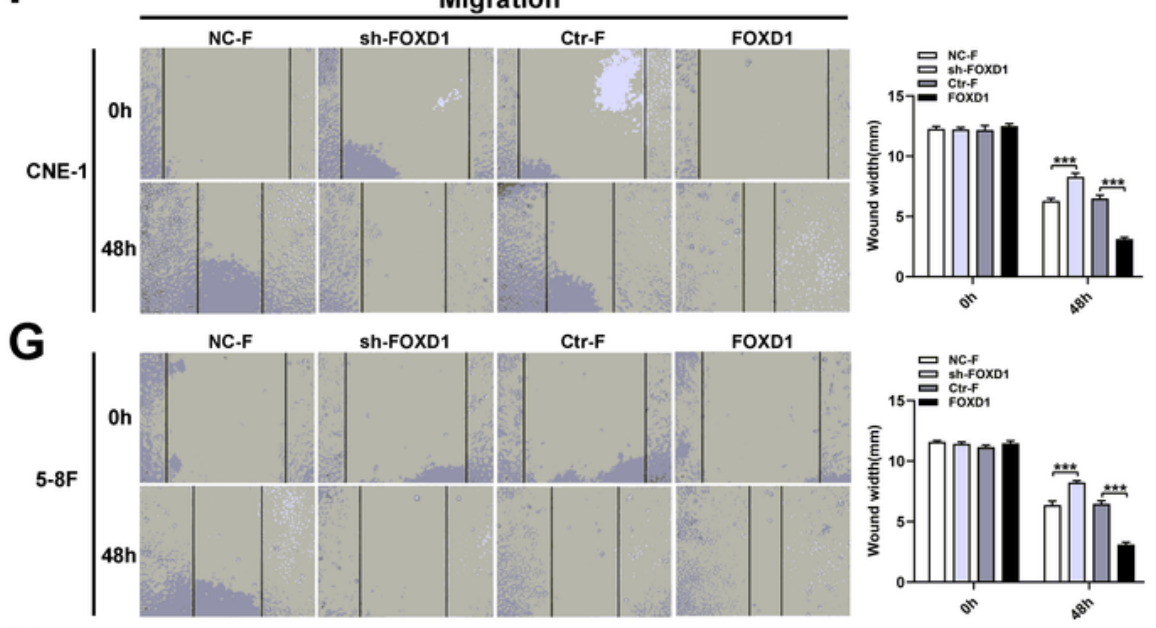

H

Invasion
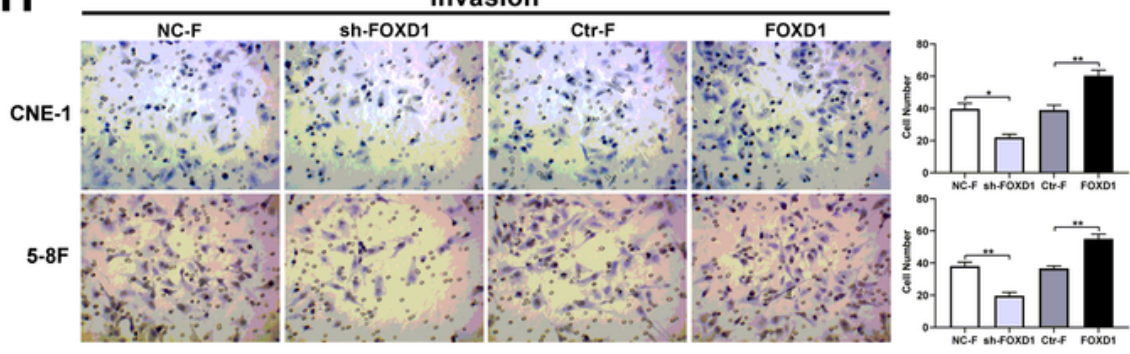

Figure 4 
FOXD1 promotes cell proliferation, migration, invasion, and induces apoptosis in NPC cells. (A) CNE-1 and 5-8F cells were transfected with FOXD1-target shRNA and FOXD1 scramble control shRNA (NC-F), respectively, and the expression level of FOXD1 was detected by qRT-PCR. (B) CNE-1 and 5-8F cells were transfected with FOXD1 overexpression plasmids or control (blank) plasmids (Ctr-F) for 48 hours, and qRT-PCR was used to analyze their efficiency. (C-D) MTT assay showing that FOXD1 knockdown inhibits cell growth, while FOXD1 overexpression accelerates cell growth in CNE-1 and 5-8F cells. (E) The effects of FOXD1 knockdown and the contribution of FOXD1-AS1 overexpression to the apoptosis of NPC cells were analyzed via flow cytometry assay. The migration $(F, G)$ and invasion $(H)$ abilities were significantly inhibited by FOXD1 silencing, whereas they were significantly increased by transfection of FOXD1 overexpression plasmids. Bars represent standard deviation, ns $P>0.05,{ }^{*} P<0.01,{ }^{\star *} P<0.001$, ${ }^{\star \star \star} P<$ 0.001 . 


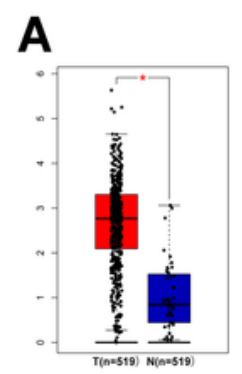

B
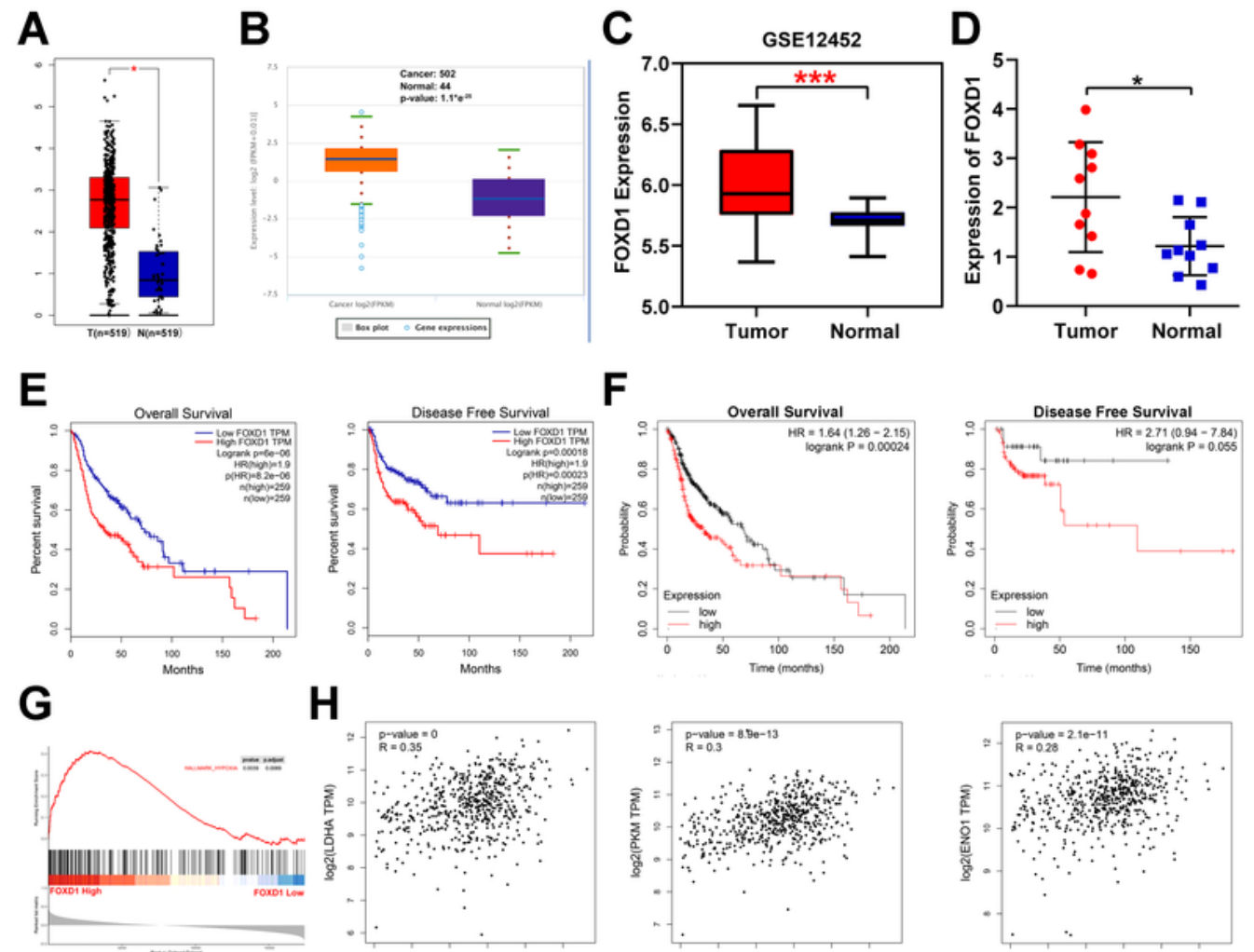

$\mathbf{H}$
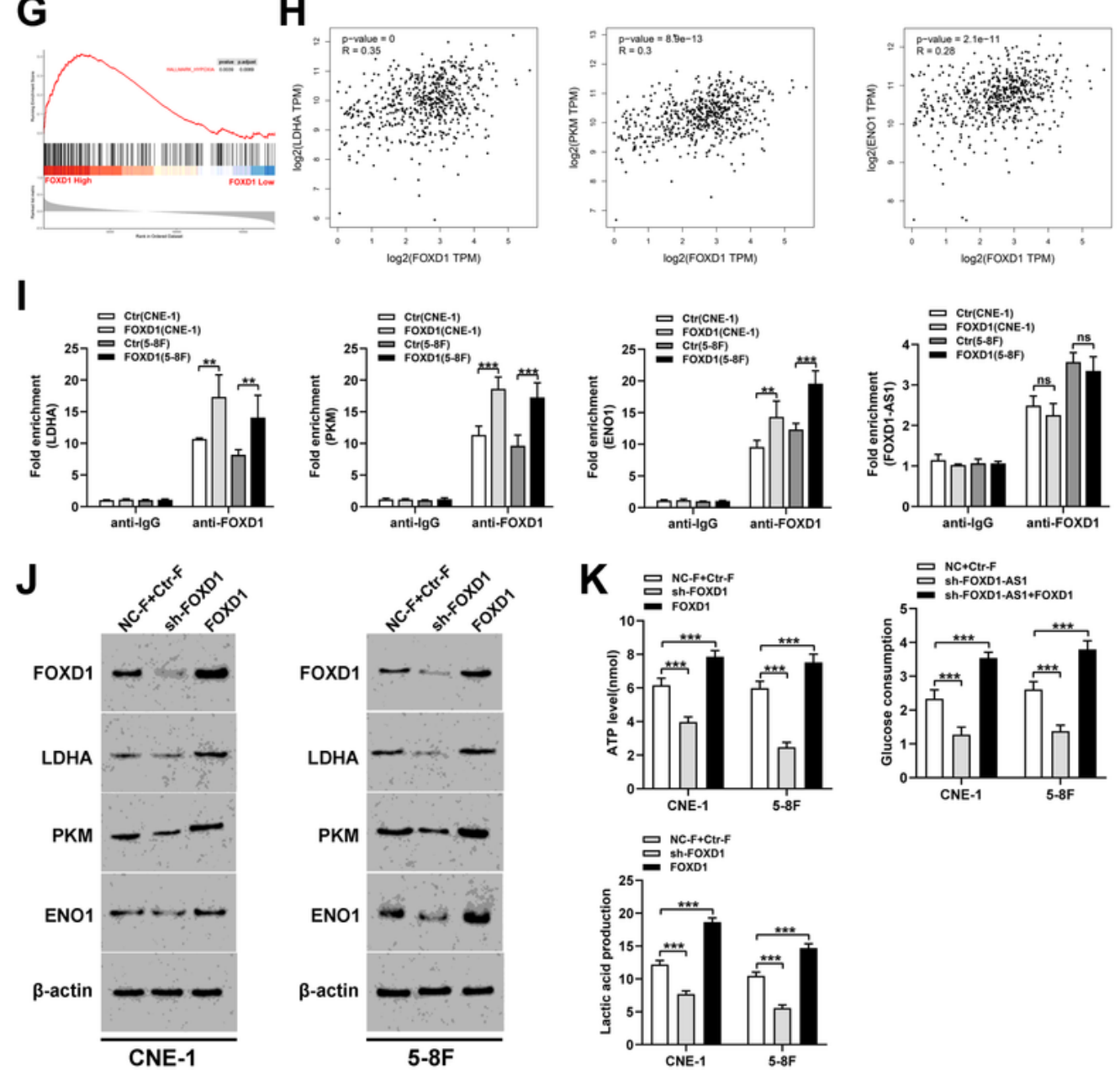

\section{Figure 5}

Upregulation of FOXD1 indicates a poor prognosis in patients with NPC and promotes the glycolysis of NPC cells. Using the GEPIA database (A), the Starbase database (B), and the GSE12452 dataset (C), we found that the expression level of FOXD1 is obviously overexpressed in tumorous tissues than in normal tissues. (D) Further qRT-PCR assay confirming that FOXD1 was upregulated in 10 pairs of NPC tissues than what was seen in adjacent normal tissues. (E) A higher expression level of FOXD1 was correlated 
with a shorter overall survival time and disease-free survival time, as seen in the GEPIA database. (F) Overall survival and disease-free survival times for FOXD1 were analyzed via a Kaplan Meier-plotter database. (G) Gene Set Enrichment Analysis (GSEA) exhibiting that FOXD1 is significantly associated with hypoxia. (H) FOXD1 expression is positively correlated with glycolysis-related genes including LDHA, PKM and ENO1. (I) ChIP assay confirms that the enrichment of LDHA, PKM, and ENO1 promoters increases obviously after overexpression of FOXD1, but upregulation of FOXD1 has no effect on the enrichment of FOXD1-AS1 promoters. (J) FOXD1 knockdown induces the protein level of LDHA, PKM, and EN01, while FOXD1 overexpression increases the expression level of these genes in CNE-1 and 5-8F cells. (K) FOXD1 knockdown decreased ATP levels, glucose consumption, and lactic acid production in CNE-1 and 5-8F cells, whereas FOXD1 overexpression increased glycolytic tumor metabolism in CNE-1 and 5-8F cells. Bars represent standard deviation, $n s P>0.05,{ }^{*} P<0.01,{ }^{\star *} P<0.001,{ }^{\star \star *} P<0.001$. 

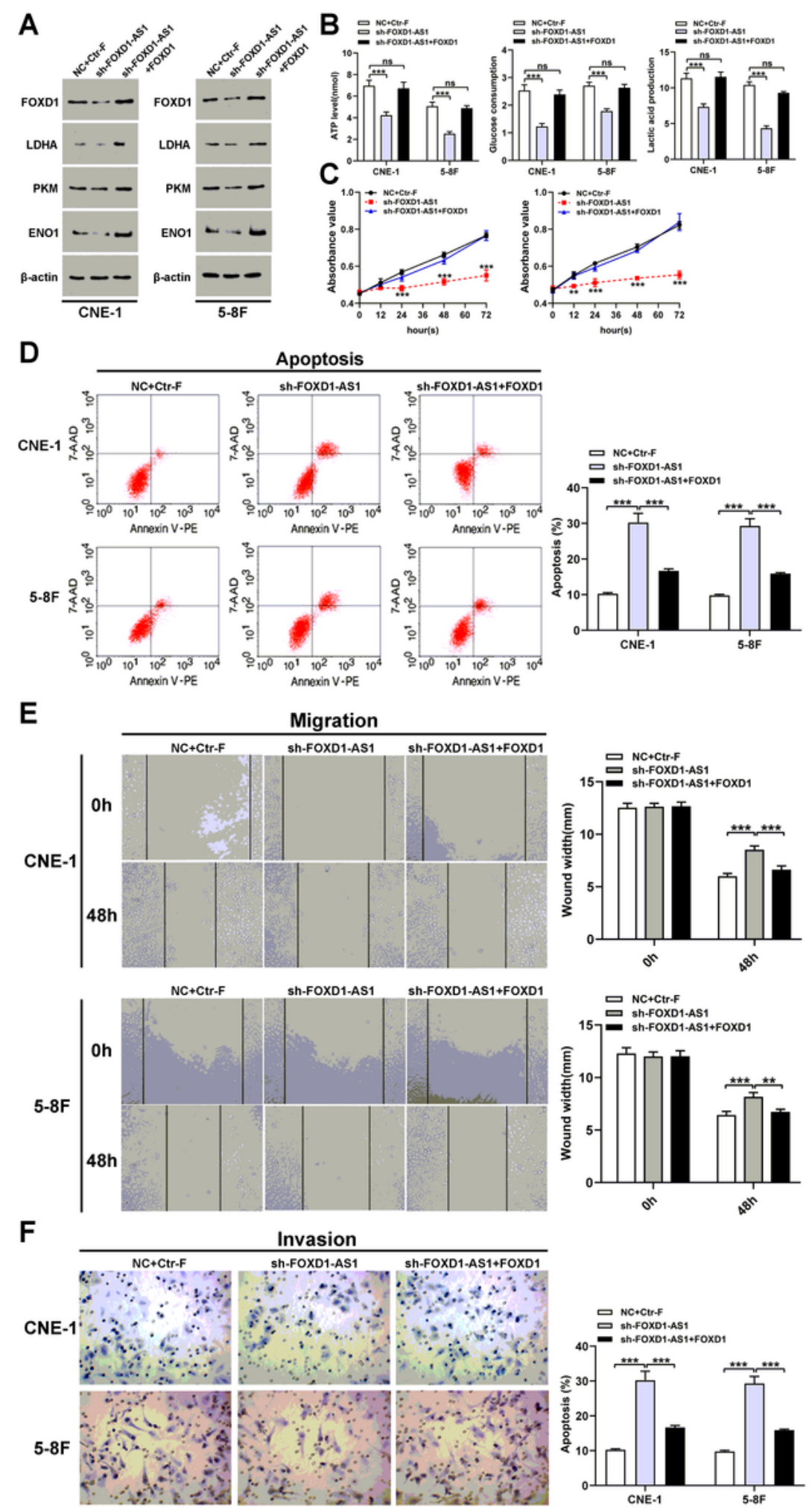

\section{Figure 6}

FOXD1-AS1 promotes progression and glycolysis by sustaining FOXD1 expression in NPC cells. (A) Knockdown of FOXD1-AS1 could decrease the protein level of FOXD1, LDHA, PKM, and ENO1, and overexpression of FOXD1 significantly reverses the effects of FOXD1-AS1 silencing in CNE-1 and 5-8F cells. Depletion of FOXD1-AS1 could induce the ATP levels, glucose consumption, lactic acid production (B), cell proliferation (C), migration (E), invasion (F), and accelerate apoptosis (D) in CNE-1 and 5-8F cells, 
whereas simultaneous overexpression of FOXD1 could reverse the effects of FOXD1-AS1 silencing. Bars represent standard deviation, ns $P>0.05, * P<0.01$, **P $<0.001$, ***P $<0.001$.
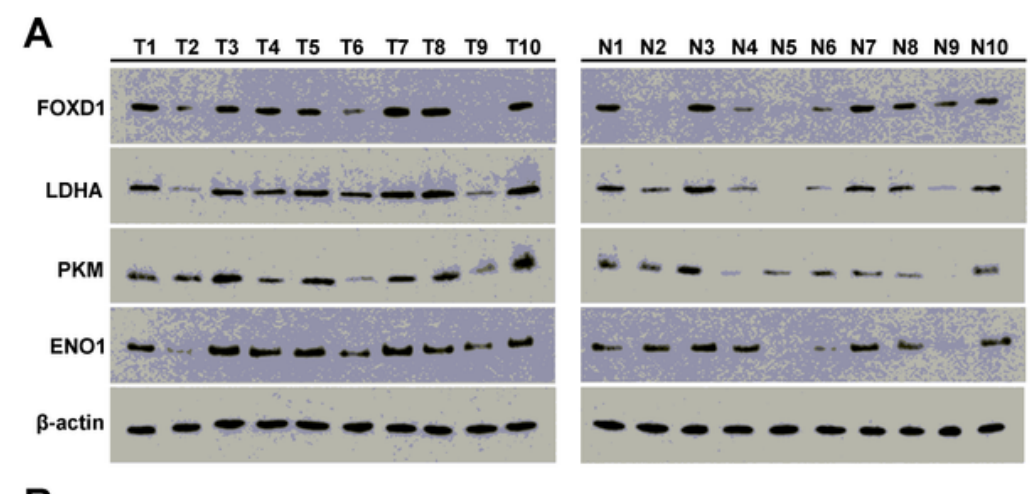

B
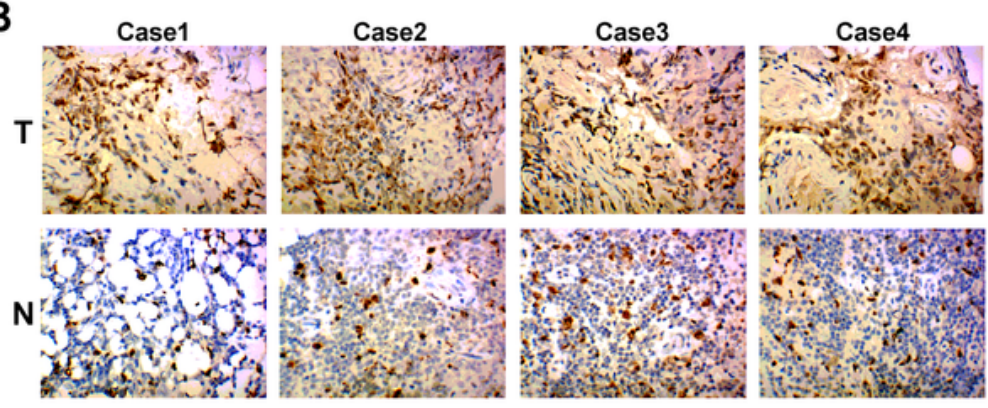

C
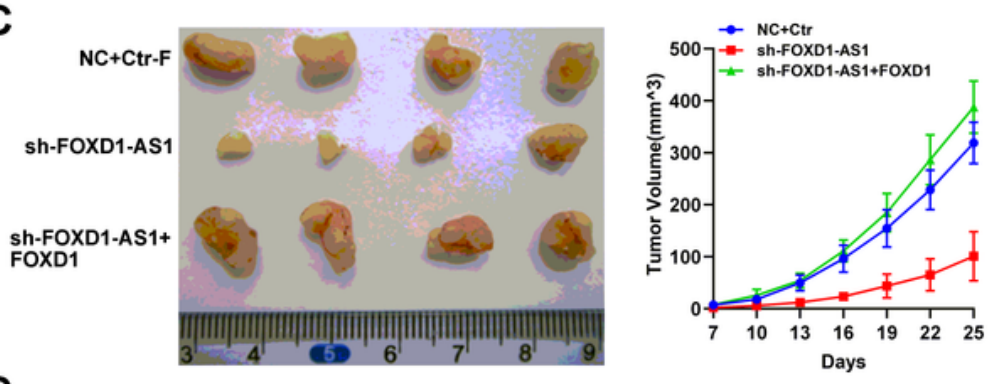

D
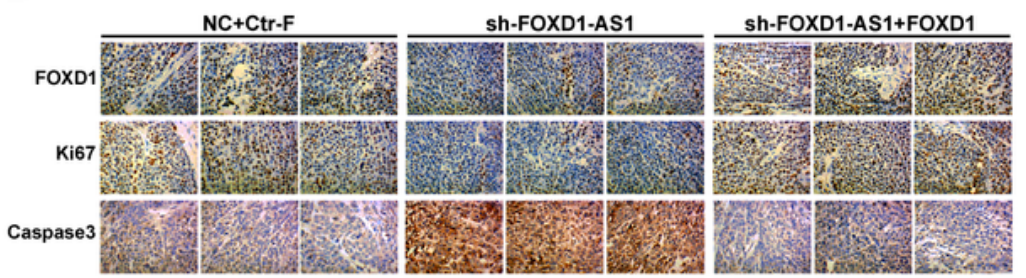

E

$\mathbf{F}$
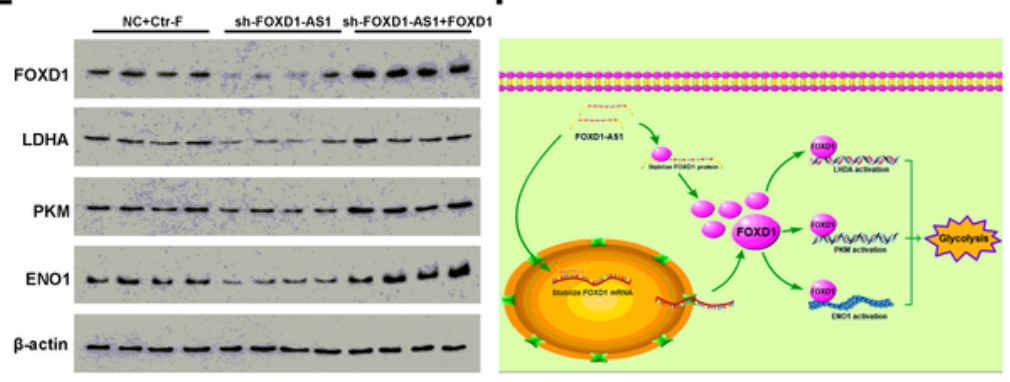

Figure 7

Effects of FOXD1-AS1/FOXD1 axis on tumor progression of NPC. (A) Western blotting analysis of FOXD1, LDHA, PKM, and ENO1 expression in 10 pairs of tumor tissues and normal tissues from patients with NPC. (B) Immunohistochemical detection of FOXD1 protein expression in tumor tissues and normal 
tissues of patients with NPC. (C) Mouse xenograft assay showing that knockdown of FOXD1-AS1 reduces the tumorigenic ability and tumor volume of CNE-1 cells, whereas FOXD1 overexpression reverses the effects of FOXD1-AS1 knockdown. (D) Immunohistochemical assay indicating that the silencing of FOXD1-AS1 decreases FOXD1, Ki67, and caspase3 protein levels in tumor tissues of mice, whereas FOXD1 overexpression reverses the protein levels of these genes. (E) Western blotting assay was performed to detect the expression level of FOXD1 and glycolysis-related proteins in the tumor tissues of mice. (F) Schematic of the present study.

\section{Supplementary Files}

This is a list of supplementary files associated with this preprint. Click to download.

- SupplementaryFigure2.tif

- SupplementaryFigure2.tif

- SupplementaryFigure1.tif

- SupplementaryFigure1.tif 